\title{
Schizophrenic patients' cognitive functions in relation to their metabolic profile: a cross-sectional, comparative study on an Egyptian sample
}

\author{
Dalia Hegazy Ali ${ }^{1 *}$ D, Doha Mostafa Elserafi ${ }^{1}$, Marwa Abdel Rahman Soltan ${ }^{1}$, Mohamed Fikry Eissa ${ }^{1}$,
} Hanan Ahmed Zein ${ }^{2}$ and Heba Hamed Elshahawi ${ }^{1}$

\begin{abstract}
Background: Patients with schizophrenia suffer from diffuse cognitive impairment and high prevalence of cardiovascular metabolic risks, associated with poor clinical outcomes. We aimed in this study to test the presence of cognitive impairment in a sample of patients with schizophrenia, and evaluate its possible relations to patients' metabolic profile. We recruited forty patients diagnosed with schizophrenia and their matched controls from the inpatient departments and outpatient services from January to December 2016. Schizophrenia diagnosis was confirmed by the ICD10 criteria checklist. Symptoms profile and severity were assessed by the Positive and Negative Syndrome Scale. Cognitive profile was assessed through (1) Trail Making Test, Parts A and B and (2) Wechsler Memory Scale-Revised Visual Reproduction Test. Metabolic profile was assessed by measuring the body mass index, fasting blood glucose, and lipid profile. SPSS (V. 22.0, IBM Corp., USA, 2013) was used for data analysis.

Results: The patients group had a significantly higher means in the speed of processing, executive function, attention, and working memory scores on TMT-A $(p=0.0)$, TMT-B $(p=0.00)$, and WMS-R $(p=0.029)$ and significantly higher FBG levels $(p=0.00)$. Correlation studies showed that the increase in patients' age, illness duration, treatments, number of hospitalizations, number of episodes and of ECT sessions received, symptoms severity, and deficits in cognitive function scores was associated with higher BMI and FBG.

Conclusions: Patients with schizophrenia have a higher prevalence of cognitive impairment and vascular risk factors than the general population. Close monitoring and early management of these risk factors can promote better cognitive abilities and overall functions.
\end{abstract}

Keyword: Schizophrenia, Cognitive impairment, Metabolic syndrome, Vascular risk

\section{Background}

Patients with schizophrenia have long been known to suffer from diffuse cognitive impairments affecting their memory; immediate and delayed recall, verbal and spatial; attention processes; executive functions; sequencing; organization flexibility; and slowed cognitive speed.

\footnotetext{
* Correspondence: daliahegazy74@yahoo.com

'Faculty of Medicine, Institute of Psychiatry, Ain Shams University, Cairo, Egypt

Full list of author information is available at the end of the article
}

Various authors have concluded that schizophrenia had cognitive profile test results, indicative of dysfunction in frontal and temporal lobes, left or right hemisphere, and basal ganglia [1]. Others have reported more widespread neuropsychological dysfunction with an average that can reach two standard deviations below the healthy controls mean [2].

These deficits are thought to be strongly related to poor clinical outcomes, perhaps more than positive and negative symptoms impact $[1,3]$. Cognitive deficits can 
be manifested as an inability to accurately recognize social cues or to respond properly to them, affecting patients' social and interpersonal skills all with their functional integration in the environment [4]. Moreover, patients' improvement in their work performance in a 6month work rehabilitation program could be predicted by their baseline performance on various cognitive tests performed [5, 6].

Unfortunately, schizophrenic patients suffer from marked and severe cognitive impairment either being on or off medication. Neuroleptic and anticholinergic naive patients still have cognitive decline [7]. Nevertheless, some psychotropic medications have cardiometabolic deleterious effects, which can affect the cognitive functions of the patients.

Some research groups reported that the presence of hypertension and elevated body mass index (BMI) in patients with schizophrenia and healthy controls was associated with poorer verbal memory performance [8]. Figures reflected the high prevalence rates of cardiovascular risks as hypertension (32.8\%), diabetes mellitus (10.9\%), dyslipidemia (47.3\%), and under-treatment of hypertension and dyslipidemia, in the schizophrenia population compared with the general population [9]. In addition, Van den Berg et al. [10] confirmed the negative effect of obesity on non-psychiatric subjects' cognitive functions as well.

Cognition impairment in this population either as a core feature of the disorder or as a complication from the associated cardiovascular risks can predict difficulties in real-world community functioning, independent residential status, and the ability to perform everyday living skills in assessment settings [11].

Understanding patients' cognitive impairment in relation to these risk factors helps in the improvement of the medical services delivered to this population, which can lead to better prognosis on clinical and functional levels and less cost and stigma. Thus, this study aimed to evaluate the cognitive functions in a sample of patients with schizophrenia and to test the presence and associations of metabolic syndrome in relation to patients' cognitive functions.

\section{Methods}

\section{Study design and setting}

The present study is a cross-sectional comparative study that was conducted between January 2016 and December 2016. Forty patients diagnosed with schizophrenia according to ICD10 were recruited from the inpatient departments and outpatient clinics in the institute of Psychiatry, Ain Shams University, and Abbassia Mental Health Hospital, Cairo, Egypt.

\section{Ethical considerations}

The study was approved by the Ethics Research Committee of the Institute of Psychiatry, Ain Shams University, and Abbassia Mental Health Hospital. An informed written consent was signed from the participants or their legal guardians, after discussing the aim and procedures of the study. Participation was totally free and voluntary. Moreover, our team clarified that patients have the right to withdraw from the study at any point without justification or negative consequences. They were informed that the results could be used for scientific publication, but their identities would be confidential. The study is consistent with the Declaration of Helsinki and World Health Organization guidelines. The reference number is not available.

Patients were illegible for the study if their illness duration exceeds 5 years, their age was above 35 years old, they received at least 6 years of education, and their Positive and Negative Syndrome Scale [PANSS] total score was less or equal to 60 . Both genders were included, and the majority of patients were only on one oral antipsychotic medication.

We excluded patients with (1) serious neurologic disorders that might affect their cognitive functions (i.e., major head injury, cerebrovascular disease, Parkinson's disease, Alzheimer's disease, seizure disorder); (2) chronic unstable medical condition, e.g., poorly controlled diabetes, hypertension, and symptomatic coronary artery disease; (3) BMI $\geq 45 \mathrm{~kg} / \mathrm{m}^{2}$; (4) severe visual or hearing impairment; (5) substance abuse or dependence in the past 3 months; and (6) pregnant nursing females or history of pregnancy in the past 12 months.

\section{Procedure}

A trained research team on data collection and standardized test administration with high inter-rater reliability collected data from the candidates' medical history (with special attention to any related past medical or neurological condition, accidents, and injuries); underwent neurological examination (when needed); administered comprehensive psychiatric assessment using the Institute of Psychiatry, Ain Shams University Hospital (ASUIP) semi-structured clinical assessment sheet, ICD10 criteria checklist for confirmation of diagnosis [12], Positive and Negative Syndrome Scale (PANSS) for the assessment of symptoms severity and profile [13]; and performed a cognitive assessment for the patients through the application of a cognitive battery. The battery included (1) Trail Making Test Parts A and B that measure the processing speed and executive function, respectively. Patients' performance was measured by the time to complete each test [14] and (2) Wechsler Memory Scale-Revised Visual Reproduction Test that measures visual learning and memory. It requires the subject 
to copy and recall abstract line drawings; the immediate recall raw score was used as the performance measure [15]. The performance measures were grouped into 4 domains: processing speed (Digit Symbol; Trail Making Test Part A), attention and working memory (Digit Span total), executive functioning (Trail Making Test Part B; WCST preservative errors and categories completed), and visual memory (visual reproduction).

Candidates' metabolic profile was assessed by measuring their body mass index, fasting blood glucose, and lipid profile. We divided the participants' weight in kilograms by height in meters squared $\left(\mathrm{kg} / \mathrm{m}^{2}\right)$ to calculate BMI. The World Health Organization standard classification for body mass index for subjects defined weights $<18.5 \mathrm{~kg} / \mathrm{m}^{2}$ as underweight, $18.5-25$ $\mathrm{kg} / \mathrm{m}^{2}$ as normal weights, $25.1-30 \mathrm{~kg} / \mathrm{m}^{2}$ as overweight, $30.1-35 \mathrm{~kg} / \mathrm{m}^{2}$ as moderately obese, and $35.1-$ $40 \mathrm{~kg} / \mathrm{m}^{2}$ as severely obese [15].

\section{Study size}

A convenient sampling technique was used. Sample size was calculated using EPI info and based on the institute registry rates for inpatient and outpatient services of two clinics/week. Considering a power of study of 0.8 at $95 \%$ confidence interval (CI), the calculated sample size was 40 patients. We included 50 patients to compensate for possible missing data. However, in the presence of drop rates, 40 patients eventually comprised our patients group.

\section{Statistical methods}

We used SPSS statistics (V. 22.0, IBM Corp., USA, 2013) for data analysis. Collected data were expressed as mean $\pm \mathrm{SD}$ for quantitative parametric measures in addition to both number and percentage for categorized data. The multiple comparisons (post hoc test or least significant difference (LSD)) followed to investigate the possible statistical significance between the 2 groups. Error probability at 0.05 was considered significant, while at 0.01 and 0.001 was highly significant. Tests can be detailed as follows: (1) comparison between two independent mean groups for parametric data using the Student $t$ test; (2) comparison between more than 2 patient groups for parametric data using the analysis of variance (ANOVA); (3) the Pearson correlation test to study the possible association between two variables among each group for parametric data; and (4) chi-square test to study the association between 2 variables or comparison between 2 independent groups as regards the categorized data.

\section{Results}

This study included 50 patients, 10 patients did not complete all the need assessments due to time arrangement difficulties, and their data were excluded. The final sample included 40 patients diagnosed as schizophrenic matched with 20 healthy controls. The patients and the control group were matching regarding, age, gender, marital status, education, occupation, smoking, and medical morbidities ( $p$ more than 0.05 )

\section{Prevalence and degree of cognitive impairment in the studied groups}

All the schizophrenic patients showed cognitive impairments. Significant differences were noted between the case and control group: in the speed of processing and executive function, attention, and working memory scores, TMT-A $(p=0.0)$, TMT-B $(p=0.00)$, and WMS$\mathrm{R}(p=0.029)$. There was no significant difference in the two groups' visual memory scores (Table 1).

\section{Patient control metabolic profile comparative data}

There was no significant difference between the case and control group BMI, but the patient group had higher means. There was a significant difference between the two groups' FBG levels $(p=0.00)$ (Table 1$)$.

\section{Correlations of patients' metabolic and cognitive profiles}

Coefficient analysis data showed that the increase in patients' age, duration of illness and treatments, number of hospitalizations, number of episodes, number of ECT sessions received, symptom severity, and deficits in cognitive function scales, on one hand, was significantly associated with higher BMI and FBG scores. However, no detected correlation between these clinical data and patients' cholesterol levels (Table 2).

Correlation coefficient analysis results showed that patients with high BMI and FBG took more time in the test performance thus had abnormal higher (TMT-A, TMT-B, WMS-R) scores. This was not noted in cases of high cholesterol levels (Table 3).

\section{Multiple regression analysis of patients data}

The increase in FBG levels was the most significantly involved predictor factor in the increase of TMT-A score, followed by patients' BMI. Patients' age, age at onset of disease, and duration of illness were all less significant (Table $4(\mathrm{~A})$ ).

The increase in FBG levels was the most significantly involved predictor factor in the increase of TMT-B and WMS-R scores. On the other hand, any increase in BMI was not associated with a significant increase in TMT-B and WMS-R scores (Table 4 (B and C)).

\section{Discussion}

The current study is a cross-sectional descriptive comparative study conducted in the institute of psychiatry Ain Shams University and Abbassia Mental Health Hospital from which a convenient sample of 60 subjects (40 
Table 1 Cognitive function and metabolic profile of the case and control groups

\begin{tabular}{|c|c|c|c|c|}
\hline & Patient $(n=40)($ mean \pm SD $)$ & Control $(n=20)($ mean \pm SD $)$ & $T$ & $p$ \\
\hline BMl & $29.01 \pm 6.2663$ & $26.675 \pm 5.4781$ & -1.482 & 0.146 \\
\hline FBG & $142.628 \pm 29.647$ & $95.95 \pm 13.9311$ & -8.293 & $0^{*}$ \\
\hline TMT-A & $100.45 \pm 17.7258$ & $62.55 \pm 9.725$ & -10.684 & $0^{*}$ \\
\hline TMT-B & $297.65 \pm 43.9933$ & $153.1 \pm 20.5347$ & -17.343 & $0^{*}$ \\
\hline WMS-R & $10.325 \pm 4,8006$ & $8 \pm 3.1623$ & -2.241 & $0.029^{*}$ \\
\hline
\end{tabular}

$B M I$ body mass index, FBG fasting blood glucose, TMT-A trail make test type A, TMT-B trail make test type B, WMS- $R$ Wechsler Memory Scale-Revised Visual Reproduction

*Significant

cases and 20 control healthy subjects) fulfilling the inclusion criteria were recruited.

The present study aimed to evaluate the cognitive functions in a sample of patients with schizophrenia and test how metabolic syndrome can be a possible predictive risk for patients' cognitive impairment.

Our results showed a significant difference between cases and controls' cognitive functions and metabolic profile data. The case group had lower cognitive functions (TMT-A, TMT-B, WMS-R score) than controls. Similarly, patients with schizophrenia had a higher prevalence of high body mass index and high blood glucose levels compared to the control group. Despite the narrow differences between the groups' BMI, however, the average percentage of obesity (BMI is $\geq 30$ ) in schizophrenic patients was more than in the controls (BMI was between 25 and 30).

According to the previous researches, as many as $80 \%$ of patients with schizophrenia had cognitive dysfunctions, possibly as a core feature of the disease [16].

These results showed that all cases in the patients group had cognitive impairments. Those impairments were significantly higher than the controls, namely in the speed of processing and executive function, attention, and working memory scores TMT-A $(p=0.0)$, TMT-B $(p=0.00)$, and WMS-R $(p=0.029)$. On the other hand, there was no significant difference between

Table 2 Correlations of patients' group metabolic profile and clinical data

\begin{tabular}{|c|c|c|c|c|c|c|c|c|c|}
\hline & \multicolumn{3}{|l|}{ BMI } & \multicolumn{3}{|l|}{ FBG } & \multicolumn{3}{|c|}{ Cholesterol level } \\
\hline & $R$ & $p$ & $\overline{\text { Sig }}$ & $R$ & $p$ & $\overline{\text { Sig }}$ & $R$ & $p$ & Sig \\
\hline Age & 0.972 & $0^{*}$ & $\mathrm{~S}$ & 0.95 & $0^{*}$ & $S$ & 0.112 & 0.857 & NS \\
\hline Age-onset & 0.93 & $0^{*}$ & S & 0.872 & $0^{*}$ & S & 0.423 & 0.478 & NS \\
\hline Dur-treat & 0.97 & $0^{*}$ & S & 0.955 & $0^{*}$ & $\mathrm{~S}$ & 0.204 & 0.742 & NS \\
\hline PANSS & 0.985 & $0^{*}$ & $\mathrm{~S}$ & 0.961 & $0^{*}$ & S & 0.427 & 0.473 & NS \\
\hline No-Hospit & 0.917 & $0^{*}$ & S & 0.898 & $0^{*}$ & $\mathrm{HS}$ & 0.346 & 0.569 & NS \\
\hline No-Epis & 0.919 & $0^{*}$ & S & 0.903 & $0^{*}$ & S & $x$ & $x$ & $x$ \\
\hline No-ECT & 0.952 & $0^{*}$ & S & 0.923 & $0^{*}$ & $\mathrm{~S}$ & 0.346 & 0.569 & NS \\
\hline
\end{tabular}

Dur-treat duration of illness, PANSS Positive and Negative Symptom Scale, NoHospit number of hospitalization, No-Epis number of episodes, No-ECT number of electroconvulsive therapy, BMI body mass index, Sig significant, NS non-significant

*Significant the groups in the visual memory scores as shown in Table 1. This matched the study in a UK Biobank data which investigated the cognitive functions footprint of different psychiatric and neurological disorders in 2017 [17]. It revealed that $1.89-3.92 \%$ of the examined sample $(n=502,642)$ who were diagnosed as schizophrenic suffered from cognitive dysfunction in reasoning, pair matching, numeric memory, and reaction time [17].

Interestingly, the current data did not show a significant difference between case and control BMI. However, there was a significant difference between cases and control FBG levels $(p=0.00)$ as shown in Table 3, which matched Pillinger et al.'s [18] results, emphasizing on the fact that glucose homeostasis is altered in schizophrenic patients, indicating a high risk for diabetes in this population. Similarly, Kato and colleagues [19] reported that the body mass index does not differ significantly between schizophrenic and non-schizophrenic patients. On the contrary, Subramanian and colleagues [20] demonstrated that patients with schizophrenia were more prone to higher body weights than the healthy controls, attributing this difference to longer duration of illness, types of medication use, and presence of a comorbid medical condition.

Table 3 Correlation between patients' cognitive functions and clinical data

\begin{tabular}{|c|c|c|c|c|c|c|c|c|c|}
\hline & \multicolumn{3}{|c|}{ TMT-A } & \multicolumn{3}{|c|}{ TMT-B } & \multicolumn{3}{|c|}{ WMS-R } \\
\hline & $R$ & $p$ & Sig & $R$ & $p$ & Sig & $R$ & $p$ & Sig \\
\hline Age & 0.974 & 0 & $\mathrm{HS}$ & 0.865 & 0 & $\mathrm{HS}$ & 0.968 & 0 & HS \\
\hline Age-onset & 0.948 & 0 & HS & 0.779 & 0 & HS & 0.973 & 0 & $\mathrm{HS}$ \\
\hline Dur-treat & 0.966 & 0 & HS & 0.891 & 0 & HS & 0.941 & 0 & $\mathrm{HS}$ \\
\hline No-Hospit & 0.943 & 0 & HS & 0.821 & 0 & HS & 0.955 & 0 & $\mathrm{HS}$ \\
\hline No-Epis & 0.912 & 0 & HS & 0.798 & 0 & HS & 0.915 & 0 & HS \\
\hline No-ECT & 0.951 & 0 & HS & 0.838 & 0 & HS & 0.961 & 0 & HS \\
\hline BMI & 0.987 & 0 & HS & 0.907 & 0 & HS & 0.97 & 0 & $\mathrm{HS}$ \\
\hline FBG & 0.967 & 0 & HS & 0.954 & 0 & HS & 0.941 & 0 & HS \\
\hline Cholesterol & 0.405 & 0.498 & NS & 0.224 & 0.718 & NS & 0.454 & 0.442 & NS \\
\hline
\end{tabular}

TMT-A trail make test type A, TMT-B trail make test type B, No-Hospit number of hospitalization, No-Epis number of episodes, No-ECT number of electroconvulsive therapy, WMS-R Wechsler Memory Scale-Visual Reproduction, $B M I$ body mass index, Chol cholesterol level, HS highly significant,

Sig significant

*Significant

**Highly significant 
Table 4 Multiple regression analysis data of patients' cognitive, clinical, and metabolic profiles

\begin{tabular}{|c|c|c|c|c|c|c|c|}
\hline & Reg. Coef. & $T$ & $p$ & Sig. & $f$ ratio & $p$ & Sig. \\
\hline \multicolumn{8}{|c|}{ A. TMT-A, clinical, and metabolic profile } \\
\hline Constant & 7.843 & 2.859 & 0.007 & HS & & & \\
\hline Age & -0.175 & -0.779 & 0.441 & NS & & & \\
\hline Age-onset & 1.275 & 4.283 & 0 & $\mathrm{HS}$ & 495.293 & 0 & HS \\
\hline Dur-IIness & 0.754 & 0.919 & 0.365 & NS & & & \\
\hline BMl & 1.075 & 2.939 & 0.006 & HS & & & \\
\hline FBG & 0.193 & 3.391 & 0.002 & $\mathrm{HS}$ & & & \\
\hline \multicolumn{8}{|c|}{ B. TMT-B, clinical, and metabolic profile } \\
\hline Constant & 111.431 & 7.147 & 0 & HS & & & \\
\hline Age & -2.169 & -1.697 & 0.099 & NS & & & \\
\hline Age-onset & -0.245 & -0.145 & 0.886 & NS & 88.964 & 0 & HS \\
\hline Dur-IIness & 2.026 & 0.434 & 0.667 & NS & & & \\
\hline BMl & 0.403 & 0.194 & 0.847 & NS & & & \\
\hline FBG & 0.909 & 5.9 & 0 & $\mathrm{HS}$ & & & \\
\hline \multicolumn{8}{|c|}{ C. WMS-R, clinical, and metabolic profile } \\
\hline Constant & -16.594 & -20.56 & 0 & $\mathrm{HS}$ & & & \\
\hline Age & -0.036 & -0.551 & 0.586 & NS & & & \\
\hline Age-onset & 0.672 & 7.671 & 0 & NS & & & \\
\hline Dur-IIness & -0.195 & -0.81 & 0.424 & NS & 418.67 & 0 & HS \\
\hline BMl & 0.152 & 1.415 & 0.166 & NS & & & \\
\hline FBG & 0.057 & 3.403 & 0.002 & HS & & & \\
\hline
\end{tabular}

Dur-IIIness duration of illness, $B M I$ body mass index, $F B G$ fasting blood glucose, TMT-A trail make test type A, TMT-B trail make test type B, WMS- $R$ Wechsler Memory Scale-Visual Reproduction

This study found that the increase in patients' BMI and FBG and not cholesterol level was associated with a decrease in candidates' cognitive functions. This came in agreement with Guo and colleagues' study [21] where they reported a significant correlation between the schizophrenic patients high BMI and low TMT-A, TMT-B, WMS-R Visual Reproduction subscale, and the WAIS Digit Symbol subscale scores $(p \leq 0.004)$ and was further confirmed by Hidese et al.'s study [22].

On the contrary, to some of our findings, some studies reported that hypertension followed by dyslipidemia were the most common vascular risks in schizophrenic patients and that increased body weight and elevated blood glucose were the least detected abnormality [19].

These study results found that high FBG followed by high BMI was the most involved factors in patients' cognitive decline (TMT-A), while age of the patient, age of onset of disease, and duration of illness were the least significant factors.

In agreement with our results, Wysokiński and colleagues [23] concluded that dyslipidemia, raised LDL, and raised blood glucose levels were the best predictors of a more severe clinical and lower cognitive abilities (worse cognitive flexibility, executive functions, complex attention composite memory, verbal memory; slower reaction time; and worse performance) in schizophrenic patients. Also, Mosiołek's [24] results which suggested that schizophrenic patients' poor performance on the cognitive tasks compared to the healthy individuals occurs early in the course of the disease, yet stabilize across the age group.

\section{Limitations and strength}

This study is one of the rare studies testing the negative effects of the prevalent metabolic disorders in the schizophrenic population on their cognitive abilities. We used objective measures in testing patients' cognitive abilities. Yet, the use of more scales in measuring patients' cognitive abilities and other metabolic profile detectors such as waist circumference would have been more elaborative. In this study, we did not measure the levels of glycosylated hemoglobin, for logistic reasons, leaving us with nonconclusive data of the long-term effect of schizophrenia on glucose blood level control. Also, we did not measure the levels of cholesterol, triglyceride, and lipoprotein in healthy controls for limited financial resources. Moreover, we could not specify from history the main cause of diabetes or high fasting glucose level, leaving antipsychotic treatments, unhealthy lifestyle, and other factors as possible causes. Further longitudinal or a cross-sectional study with a larger number of cases from different hospitals in addition to bypassing the methodological limitations would have helped in the result generalization.

\section{Conclusions}

Patients with schizophrenia had a higher prevalence of cognitive impairment and vascular risk factors than the general population. The increase of body mass index and fasting blood glucose levels were associated with longer duration of illness and higher symptoms severity and with more decline in patients' cognitive abilities. The increase in patients' age, number of hospitalizations, episodes of psychosis, and number of ECT sessions was associated with deterioration of patients' cognitive functions. Close monitoring and early management of these risk factors in addition to many others all promoting a healthy lifestyle for schizophrenic patients can improve their cognitive abilities and the overall functions.

\section{Abbreviations}

BMI: Body mass index; FBG: Fasting blood glucose; TMT-A: Trail make test type A; TMT-B: Trail make test type B; WMS-R: Wechsler Memory Scale-

Revised Visual Reproduction; PANSS: Positive and Negative Symptom Scale; ECT: Electroconvulsive therapy; ICD10: International Classification of Disease 10th revision 


\section{Acknowledgements}

We wish to thank the entire participants who gave us a hand to accomplish this research which we consider it as one of the few Egyptian work out discussing the metabolic profile effect on patients' cognitive functions especially those suffering from schizophrenia. Special thanks goes to Youssef wael ahmed Mohamed zohdy for the effort in data entry for all cases.

\section{Authors' contributions}

MA, DH, and DS analyzed the data and interpreted it. HS and MF suggested the topic and have shared in the methodology and interpretation. $\mathrm{DH}$ and HA searched for the resources and were the main contributors in writing the manuscript. All authors read and approved the final manuscript for submission.

\section{Funding}

None

\section{Availability of data and materials}

Not applicable

\section{Ethics approval and consent to participate}

The study protocol was approved by the Ethics and Research Committee of the Institute of Psychiatry, Faculty of Medicine, Ain Shams University, and Abbassia Mental Health Hospital. All participants or their legal guardians signed written informed consent, after discussing the aim and procedures of the study. Participation was free and voluntary. The study is consistent with the Good Clinical Practices, the Declaration of Helsinki, and the World Health Organization Guidelines. The reference number is not available.

\section{Consent for publication}

Not applicable

\section{Competing interests}

The authors declare that they have no competing interests.

\section{Author details}

'Faculty of Medicine, Institute of Psychiatry, Ain Shams University, Cairo,

Egypt. ${ }^{2}$ Abbassia Mental Health Hospital, Cairo, Egypt.

Received: 13 May 2020 Accepted: 31 August 2020

Published online: 20 October 2020

\section{References}

1. Hoff AL, Kremen WS (2003) Neuropsychology in schizophrenia: an update. Current Opinion in Psychiatry 16:149-155

2. Keefe RS, Buchanan RW, Marder SR, Schooler NR, Dugar A, Zivkov M, Stewart M(2011a). Characteristics of the MATRICS Consensus Cognitive Battery in a 29-site antipsychotic schizophrenia clinical trial. Schizophr Res Feb,125(2-3):161-168.

3. Krishnan RR, Fivaz M, Kraus MS, Keefe RS (2011). Hierarchical temporal processing deficit model of reality distortion and psychoses. Mol Psychiatry Feb,16(2):129-144.

4. Y. Vishnu Gopal \& Hannele Variend (2005). First-episode schizophrenia: review of cognitive deficits and cognitive remediation. Adv Psychiatr Treat 11: $38-44$

5. McGurk SR, Mueser KT, Harvey PD, LaPuglia R, Marder J (2003) Cognitive and symptom predictors of work outcomes for clients with schizophrenia in supported employment. PsychiatrServ 54:1129-1135

6. Mausbach BT, Bowie CR, Harvey PD, Twamley EW, Goldman SR, Jeste DV, Patterson TL (2008) Usefulness of the UCSD performance-based skills assessment (UPSA) for predicting residential independence in patients with chronic schizophrenia. J Psychiatr Res 42:320-327

7. Saykin AJ, Shtasel DL, Gur RE et al (1994) Neuropsychological deficits in neuroleptic naive patients with first episode schizophrenia. Archives of Psychiatry 51:124-131

8. Friedman J, Wallenstein S, Moshier E, Parrella M, White L, Bowler S, Gottlieb S, Harvey PD, McGinn TG, Flanagan L, Davis KL (2013) The effects of hypertension and body mass index on cognition in schizophrenia. Am J Psychiatry 167(10):1232-1239

9. Nasrallah HA, Meyer JM, Goff DC, McEvoy JP, Davis SM, Stroup TS, Lieberman JA (2010) Low rates of treatment for hypertension, dyslipidemia, and diabetes in schizophrenia. data from the CATIE schizophrenia tria sample at baseline. Schizophr Res 86:15-22

10. van den Berg E, Kloppenborg RP, Kessels RP, Kappelle LJ, Biessels GJ (2009) Type 2 diabetes mellitus, hypertension, dyslipidemia and obesity: a systematic comparison of their impact on cognition. BiochimBiophysActa. 1792(5):470-481

11. Evans JD, Heaton RK, Paulsen JS, Palmer BW, Patterson T, Jeste DV (2003) The relationship of neuropsychological abilities to specific domains of functional capacity in older schizophrenic patients. Biol Psychiatry 53:422-430

12. Janca A and Hiller W (1996). ICD-10 checklists a tool for clinicians' use of the ICD-10 classification of mental and behavioral disorders. Compr Psychiatry May-Jun,37(3):180-187.

13. Kay SR, Fiszbein A, Opler LA (1987) The Positive and Negative Syndrome Scale (PANSS) for schizophrenia. Schizophr Bull 13(2):261-276

14. Reitan, R. M., \& Wolfson, D. The Halstead-Reitan Neuropsychological Test Battery: theory and clinical interpretation (2nd ed.)(1993):Tucson, AZ: Neuropsychology Press.

15. Wechsler D (1987) Wechsler memory scale-revised

16. Kahn RS, Keefe RSE (2013) Schizophrenia is a cognitive illness time for a change in focus. JAMA Psychiatry 70:1107-1112

17. Cullen B, Smith DJ, Deary IJ, Evans JJ, Pell JP (2017) The 'cognitive footprint' of psychiatric and neurological conditions: cross-sectional study in the UK Biobank cohort. ActaPsychiatrScand 135:593-605

18. Pillinger T, Beck K, Gobjila C, Donocik JG, Jauhar S, Howes OD (2017). Impaired glucose homeostasis in first episode schizophrenia: a systemic review and meta-analysis. JAMA PsychiatryMar 1,74(3):261-269.

19. Kato MM, Currier MB, Villaverde O, Gonzalez-Blanco M. The relation between body fat distribution and cardiovascular risk factors in patients with schizophrenia (2005): a cross-sectional pilot study. Prim Care Companion J Clin Psychiatry 7(3):115-8; quiz 119-20. (https://www.ncbi.nlm.nih.gov/ pubmed/16027766)

20. Subramaniam M, Lam M, Guo ME, He WY, Lee J, Verma S, Chong SA. Body mass index, obesity, and psychopathology in patients with schizophrenia (2014). J Clin Psychopharmacol Feb,34(1):40-46.

21. Guo X, Zhang Z, Wei Q, Lv H, Wu R, Zhao J (2013) The relationship between obesity and neurocognitive function in Chinese patients with schizophrenia. BMC Psychiatry 13:109

22. Hidese S, Matsuo J, Ishida I, Hiraishi M, Teraishi T, Ota M, Hattori K, Kunugi H (2018). Relationship of handgrip strength and body mass index with cognitive functions in patient with schizophrenia. Front PsychiatryApr 25,9:156.

23. Wysokiński A, Dzienniak M, Kłoszewska I (2013) Effect of metabolic abnormalities on cognitive performance and clinical symptoms in schizophrenia. Arch Psychiatry Psychother 4:13-25

24. Mosiołek A, Gierus J, Koweszko T, Szulc A (2016) Cognitive impairment in schizophrenia across age groups: a case-control study. BMC Psychiatry 16 37. https://doi.org/10.1186/s12888-016-0749-1

\section{Publisher's Note}

Springer Nature remains neutral with regard to jurisdictional claims in published maps and institutional affiliations.

\section{Submit your manuscript to a SpringerOpen ${ }^{\circ}$ journal and benefit from:}

- Convenient online submission

- Rigorous peer review

- Open access: articles freely available online

- High visibility within the field

Retaining the copyright to your article

Submit your next manuscript at $>$ springeropen.com 